\title{
Digital entrepreneurship and its impact on digital supply chains: The mediating role of business intelligence applications
}

\author{
Heba Awawdeh ${ }^{a}$, Hanadi Abulaila ${ }^{a}$, Abdallah Alshanty ${ }^{a}$ and Amrou Alzoubi ${ }^{a}$
}

${ }^{\mathrm{a} A \text { Arab University collage of Technology, Jordan }}$

CH R O N I C L E ABSTRACT

Article history:

Received: June 18, 2021

Received in revised format: June 29,2021

Accepted: September 6, 2021

Available online: September 9, 2021

Keywords:

Digital Entrepreneurship

Digital supply chains

Business intelligence applications

Jordanian hotels

\begin{abstract}
This study aimed to test and evaluate the impact of Digital Entrepreneurship and its impact on digital supply chains in Jordanian hotels, and the mediating role of digital supply chains in this relationship. The descriptive analytical method was used, and the study population consisted of (835) male and female employees, and a random sample was used with a simple random sample of (342) participants. To achieve the objectives of the study, a developed questionnaire was used to collect data from the sample members. The study adopted the Statistical Package for Social Sciences (SPSS.V.22) and Structural Equations Modeling (SEM) using the AMOS program for path analysis and to perform statistical analysis using: Descriptive and inferential statistics measures, including Multiple linear regression, Pearson correlation coefficient, skew coefficient, multiple linear correlation, variance inflation coefficient and permissible variance. The study reached results, the most important of which were: The most important results of the study were: the presence of an important impact of Digital Entrepreneurship in digital supply chains and the presence of a significant impact of Digital Entrepreneurship through business intelligence applications as an intermediate variable in digital supply chains. The study recommends the need to enhance Digital Entrepreneurship in Jordanian hotels by focusing on the holistic view of these hotels and their environment, whether the internal environment that focuses on strengths and weaknesses in the hotel's capabilities or their external environment that brings opportunities and challenges.
\end{abstract}

C 2022 by the authors; licensee Growing Science, Canada.

\section{Introduction}

All business organizations in our time, whether public or private, face productivity or service. Many challenges arise because of the rapid and continuous scientific and technical changes and developments, and in the face of these hectic challenges, the traditional management, with its operations and means, is unable to make the organization capable of competition. This makes it imperative for these organizations to use all available contemporary administrative methods that enable them to do so. According to Autio et al. (2018), digital entrepreneurship helps the organization focus its vision and priorities in responding to the changes taking place in the environment around it and to ensure that the organization's members are working towards achieving the same goals. Of course, what is meant by the word "strategic" is to give the character of a long-term and comprehensive view of leadership. Digital Entrepreneurship is also concerned with the general affairs of the organization, and digital entrepreneurship begins and is directed by the highest administrative level, but all levels of management must participate in it to work. Others (Anwar, \& Daniel, 2016; Bach et al., 2018 ) indicate that digital Entrepreneurship consists of three main processes that include: digital creativity, digital leadership, and then the digital knowledge process, which includes choosing the most appropriate alternatives to management trends, the organization may choose one or several strategies and usually What each strategy has advantages and disadvantages that enable management to conduct a balancing process to

* Corresponding author.

E-mail address: hebaawadeh1984@gmail.com (H. Awawdeh)

(C) 2022 by the authors; licensee Growing Science, Canada. doi: $10.5267 /$ j.ijdns.2021.9.005 
achieve the organization's goals efficiently and effectively. Farani et al. (2017) indicate that digital entrepreneurship is linked to the organization's need for growth and survival in a constantly changing environment, so it focuses on two types of activities, the first of which is diagnosing the strengths and weaknesses of the organization, and the second is determining the strategic actions necessary to maintain and improve the level of performance. Digital Entrepreneurship leads organizations to obtain many benefits (Zhu, \& Lin, 2018). It provides the organization with a guide on what it seeks to achieve and provides officials with the style and features of the environment conditions surrounding the organization, and helps the organization anticipate changes in the surrounding environment and how to adapt with it to allocate the available resources and determine the methods of their use, organize the sequence at the different administrative levels, clarify the image of the organization in front of all stakeholder groups, and increase efficiency by increasing competition, as the entry of new competitors motivates others to respond efficiently and effectively.

The success in managing the digital supply chain is one of the strategic challenges facing the group, as its success works on the integration between the parties of the chain, which are suppliers, manufacturers, and customers. Therefore, digital supply chain management has become successful strategies to link partners together and take care of the movement of materials starting from supply sources and ending with the delivery of products to customers (Asamoah et al., 2021). Therefore, the importance of digital supply chain management stems from the need to implement it effectively, and then there are several issues that push organizations to adopt the supply chain management approach, namely: the need to improve operations, raise the level of external procurement, reduce transportation costs, and the complexity of supply chains, and then the need for effective inventory management (El Baz et al., 2019). The main objective of digital supply chain management is to meet customer requirements through optimal use of resources including distribution of capabilities, inventory, and labor (Shen et al., 2019). In theory, the supply chain seeks to link supply and demand and do so with a minimum inventory. It also includes various aspects of supply chain optimization, coordination with suppliers to eliminate bottlenecks, strategic sourcing to achieve a balance between transportation and lowest material cost and its timely implementation (Giannakis et al., 2019). The supply chain offers wonderful and diverse job opportunities in seven areas: Senior management, logistics information systems, warehousing, transportation, material control, inventory, purchasing, marketing and sales, and the work environments include distribution facilities, terminals, offices, and operations centers (Kumar, \& Singh, 2017). Business organizations are constantly working to achieve success and maintain their success and survival, and this makes them constantly seek to develop, improve and modify their strategies, to develop their products, and to use renewable tools and means. The departments of modern organizations seek to benefit from the knowledge and sciences that researchers constantly create and develop. These efforts lead to new opportunities and continuous progress and development, as business intelligence plays a vital role in the development of the strategy, especially considering the changing work environment and the challenges that accompany it. This requires decision-makers to use all intelligence techniques to face these challenges (Nithya \& Kiruthika, 2021).

Business Intelligence supports the organization with multiple patterns that are comprehensive for all its organizational, marketing, and competitive activities by providing information about the environment, working on its analysis, and taking the appropriate decision. This is supported by a deep analysis of the main competitors and their close monitoring of their actions, as well as a deep analysis of consumer positions within the market. The application of business intelligence is an important key to rationalizing decisions and increasing their efficiency, which contributes to giving a better position to the organization, especially considering the challenges and threats posed by the turbulent environment. Collins (2017) identified the importance of business intelligence by providing more accurate historical data through accurate environmental analysis that is done by predicting the future and its trends, providing decision makers with the information they need, and keeping records, sales and movements of competitors, market opportunities and competitor threats to them. Positive impact on achieving the organization's competitive advantages, expanding target markets, and increasing market share. This is done through marketing business intelligence tools and thus the intelligence contributes to the growth of the organization and its prosperity in the market. Fuld (2015) indicated that the most important benefit that the organization can derive from business intelligence is to increase its capabilities to predict the future using intelligent analysis tools, especially since forecasting is a vital part of the business intelligence process, and forecasting contributes to reducing the possibility of strategic surprises.

The commitment to applying digital entrepreneurship is a necessary model for organizations striving to enhance and adapt to the digital supply chain, and the core of management's mission has become to use logic and scientific prediction instead of chaos, as business intelligence is now seen as the primary key to the success and competitive advantage of organizations and permeates into All factors of organizational success. Therefore, this study came to know the impact of digital Entrepreneurship on digital supply chains through business intelligence applications in the Jordanian hotel sector.

\section{The study Problem}

The large and accelerating competition between organizations, which transcends regional borders, has prompted these organizations to research how to enhance the dimensions of digital entrepreneurship and digital supply chain management through the applications of business intelligence in them so that it leads to an increase in the effectiveness of business organizations and human behavior that leads to achieving excellence, exclusivity and improving potency. Digital entrepreneurship also provides an appropriate structure through which organizations can set their goals, the necessary means to achieve these goals, and monitor performance. 
The Corona crisis on the tourism sector is one of the most severe global economic crises that has afflicted the global economy since September 2008, and one of the most severe global economic crisis after the Great Depression of 1929. The seriousness and violence of this crisis comes as it revealed the fragility of the global economic system in the face of the Corona crisis, and Jordan is part of this. The world, especially since the tourism sector occupies a leading and pivotal role in the national economy of any country, and Jordan is a prominent tourist landmark in the Middle East, and it is the focus of domestic and foreign tourism for its easy access by land, sea, and air. The Jordanian hotel sector, as one of the pillars of the tourism sector, is facing fundamental changes, new trends and recent developments that we see in various fields, as it was not immune to the environment of change that has affected its survival and future, due to the poor awareness of the relationship between the dimensions of Entrepreneurship and digital supply chain management through business intelligence applications. In addition to the wave of challenges it faces, which are represented in the difficulty of strategic forecasting for its future and the mobility of its activities and objectives, which would shackle it and affect its effectiveness and performance and prevent it from reaching the level of excellence required to be achieved, due to the importance of business intelligence in making and making strategic decisions and in formulating basic strategies and to provide A base of information to meet the requirements of decision makers. Especially in that the Jordanian hotel sector strives to reach excellence in performance and maintain its continuity in a competitive environment. This is in fact due to the nature of management and the administrative approach followed, which is often due to temporary or circumstantial solutions to address the problem in a quick way, so this study comes to answer the following question: Is there a role for digital Entrepreneurship on digital supply chains through business intelligence applications The Jordanian hotel sector?

The importance of the study: The theoretical importance of this study is that it deals with one of the organizational concepts, which is the dimensions of digital Entrepreneurship, which is one of the important topics in modern management thought, as it is an important source of the organization's survival and sustainability, especially in institutions that seek to adopt the modern management strategy that It allows workers to participate in decision-making, exploit opportunities, and increase their competitiveness and creativity.

As for the practical importance of this study, it stems from the fact that it examines the role of digital entrepreneurship on digital supply chains through business intelligence applications in the Jordanian hotel sector, which helps the concerned authorities in these hotels to adopt the recommendations that this study will present in terms of information on digital entrepreneurship and supply chains. Digital and business intelligence applications, which can be used to develop sound foundations for understanding the process of digital Entrepreneurship and digital supply chains through effective business intelligence applications in Jordanian organizations, which includes the need to make a radical and total change in the way the organization performs its activities and operations; Because an organization that does not change becomes old and disappears. In addition to enabling the organization to respond to the competition of other organizations, whether within the community in which it is located or outside it.

\section{Objectives of the study}

This study aims to demonstrate the role of digital entrepreneurship on digital supply chains through business intelligence applications in the Jordanian hotel sector, by achieving the following objectives:

1. Identifying the impact of digital Entrepreneurship in its dimensions (digital creativity, digital leadership, digital knowledge) on digital supply chains through business intelligence applications in the Jordanian hotel sector.

2. Identifying the impact of digital Entrepreneurship in its dimensions (digital creativity, digital leadership, digital knowledge) on digital supply chains through business intelligence applications in the Jordanian hotel sector.

3. This study serves Jordanian organizations with what they can provide of information on digital Entrepreneurship and digital supply chains through business intelligence applications, which can be used in a serious commitment to applying digital Entrepreneurship and business intelligence in Jordanian organizations, in order to increase their effectiveness and benefit from them in programs Enhanced training for the availability of the dimensions of the role of digital supply chains and business intelligence and raising awareness among organizations' departments about the importance of digital Entrepreneurship to achieve the desired organizational goals.

\section{Study hypotheses:}

This study attempts to test the following null hypotheses:

$\mathrm{H}_{01}$ : There is no statistically significant effect for digital entrepreneurship with its dimensions (digital creativity, digital leadership, digital knowledge), in the digital supply chains with its dimensions (digital supply, digital marketing, digital services, digital logistics) in the sector Jordanian hotels.

$\mathrm{H}_{02}$ : There is no statistically significant effect of digital entrepreneurship with its dimensions (digital creativity, digital leadership, digital knowledge) on digital supply chains through business intelligence applications in the Jordanian hotel sector. 
a. Digital Entrepreneurship: A broad scope for uniting new projects and transforming existing projects and companies through the development of new digital technologies or new use of these technologies. The concept of digital entrepreneurship is linked to three variables: digital creativity, digital leadership, and digital knowledge.

b. Digital supply chains: All activities related to the flow and manufacture of products through suppliers to the final consumer in addition to the flow of information. Both flows take place in both directions from suppliers to customers and vice versa from customers to suppliers. The concept of digital supply chains is linked to four variables: digital supply, digital marketing, Digital services, digital logistics.

c. Business Intelligence: It is a set of methodologies, processes, applications and techniques, tools and methods, which are used to analyze business information and convert it into valuable information. It is the process. Business intelligence systems collect and analyze data from internal and external sources, according to the desired purposes for taking the various decisions of the organization in a way that enhances its competitive position.

Study model

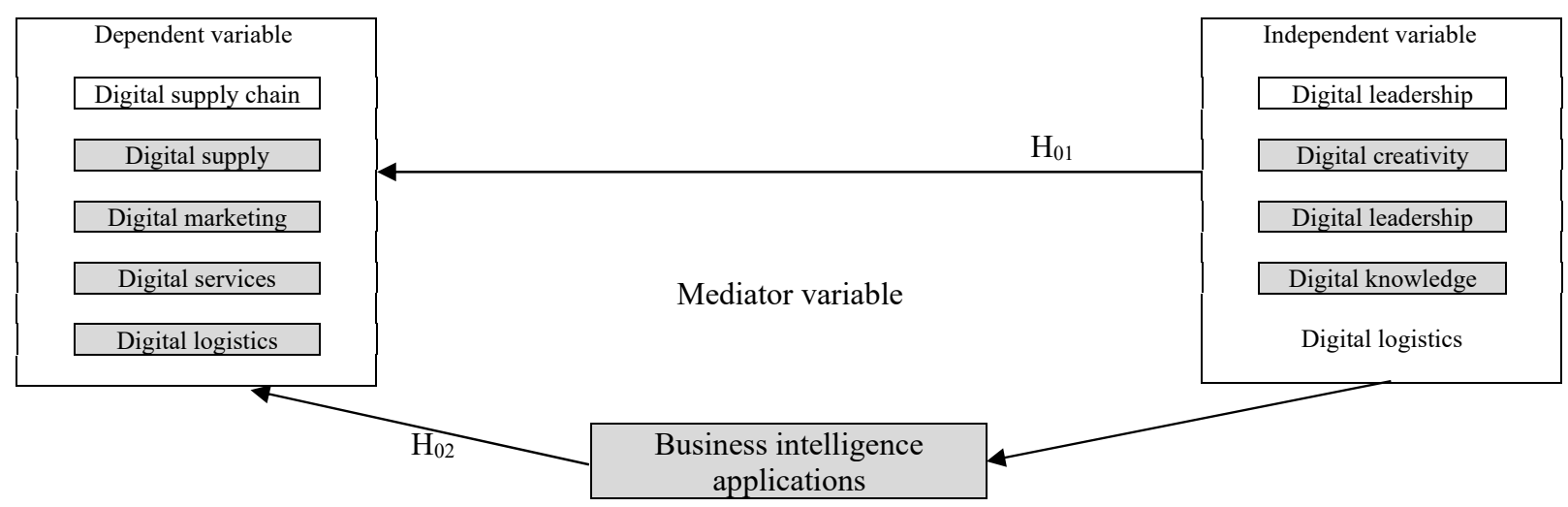

Fig. 1. The proposed study (Al-Tarawneh \& Shalash, 2015; Nsour, 2020; Al-Barashdiya et al., 2021; )

\section{Study methodology}

This study is based on the use of two scientific research methodologies:

A- Descriptive analytical approach: to review the most important literature related to the impact of digital Entrepreneurship in digital supply chains, the mediating role of business intelligence applications in the Jordanian hotel sector, as well as refer to previous studies with some analysis and comparisons whenever possible to cover the theoretical side of the study.

B - The field research method: It was used to cover the practical side of this study, through which the study attempts to answer its questions, and derive its results by relying on a questionnaire designed for the purposes of this study in accordance with the recognized practical steps.

\subsection{Study community and sample}

The target population of the study consists of all employees in hotels in the city of Amman, whose number is (835) male and female employees. A simple random sample of (50\%) was withdrawn from the study population and its size was (717) male and female employees. A simple random sample of (50\%) was withdrawn from the study population and its size was (418) male and female employees. Questionnaires were distributed to all sample items, and (357) questionnaires were recovered, i.e. $(85.4 \%)$ of the study sample size, and (15) questionnaires were excluded because they were not suitable for analysis, and thus the number of questionnaires valid for analysis became (342), forming (81.8) \%) of the study sample.

It is noted from Table (1) that with regard to gender, the highest percentage was in favor of males, reaching (60.8\%), while the percentage of females came $(39.2 \%)$.

With regard to the length of service, the workers whose experience ( 5 years or less) constituted (24.6\%), and (25.4\%) of the study population had an experience of (6-10 years), and that (15.5\%) of the study population was Their experience ranged from (11-15 years), while (34.5\%) of the study population had experience (16 years or more).

With regard to the educational qualification variable, it was found that the highest percentage of workers with a bachelor's degree was $(62.6 \%)$, followed by the percentage of workers with an intermediate diploma, which amounted to (29.2\%), and finally, the percentage of workers with postgraduate studies, which amounted to (8.2) \%). 
As for the age variable, workers whose ages ranged (from 31-40 years) constituted (41.2\%), followed by workers whose ages ranged between (41-50 years) with a percentage of (36\%), followed by workers whose ages ranged between (30 years or younger) at a rate of $(19 \%)$, and finally, workers whose age was (51 years and over) came in the last rank with a percentage of $(3.8 \%)$.

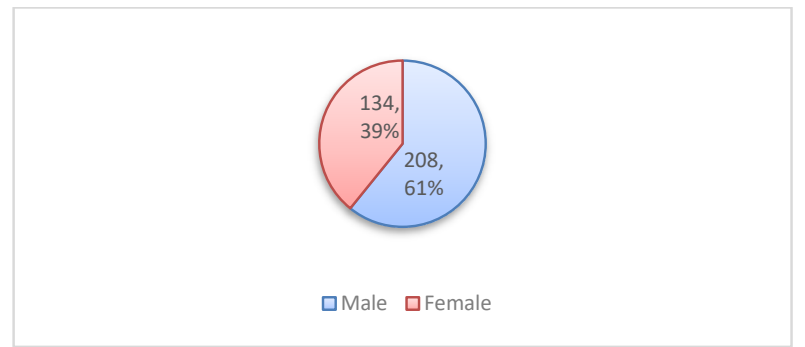

Gender

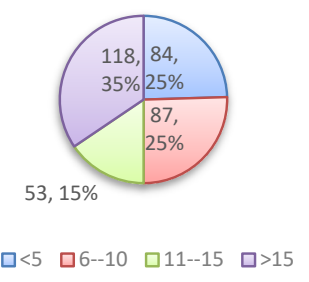

Length of service

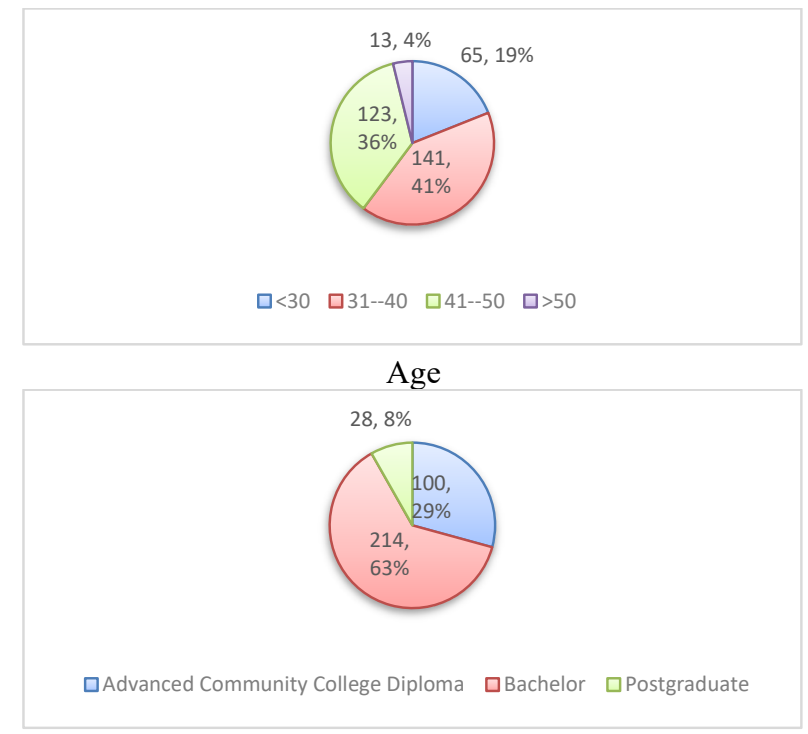

Educational background

Fig. 2. Personal characteristics of the participants

\subsection{Study tool}

A questionnaire was developed to measure the impact of digital Entrepreneurship in digital supply chains, the mediating role of business intelligence applications in the Jordanian hotel sector, and the following is an explanation of the parts of the study tool:

The first part: includes the characteristics of the study sample considering demographic or personality and occupational variables such as gender, age, educational qualification, and years of experience.

Part Two: It includes paragraphs aimed at defining the impact of digital Entrepreneurship on digital supply chains, the mediating role of business intelligence applications in the Jordanian hotel sector, as the sections for this variable were guided by studying each of (Al-Nisour, 2020) and it includes three sub-dimensions to measure digital Entrepreneurship. And represented in: (digital creativity, digital leadership, digital knowledge) and a study (Safa, 2018; Al-Tarawneh \& Shalash, 2015) was used to measure the dependent variable digital supply chains. As for business intelligence applications, a study was used (AlTamari, 2017; Younis, 2015; Al-Ghazawi, 2013).

The answers were classified according to the five-point Likert scale, and were identified with five answers: (always apply, often apply, sometimes apply, rarely apply, never apply), and the answers were given numbers from (1-5), so that the number (1) indicates ( No. (2) on (rarely applicable), number (3) on (sometimes applicable), number (4) on (often applicable), and number (5) on (always apply).

\subsection{Validity of the tool}

Table 1

The coefficient of internal consistency for each of the study variables in all its dimensions

\begin{tabular}{|c|c|c|}
\hline The tool & Dimensions & Stability Coefficient (Cronbach Alpha) \\
\hline \multirow{3}{*}{ Digital entrepreneurship } & digital creativity & 0.89 \\
\hline & digital leadership & 0.85 \\
\hline & digital knowledge & 0.81 \\
\hline \multirow{4}{*}{ Digital supply chains } & Digital supply & 0.88 \\
\hline & digital marketing & 0.91 \\
\hline & digital services & 0.87 \\
\hline & digital logistics & 0.90 \\
\hline Business intelligence applications & & 0.83 \\
\hline Total & & 0.89 \\
\hline
\end{tabular}

The questionnaire was presented to (7) arbitrators from specialized management professors and faculty members in Jordanian universities, to verify the validity of the questionnaire's paragraphs, and their comments were taken, and some paragraphs 
were reformulated, and the required modifications were made, in a precise manner that achieves a balance between the contents of the questionnaire. in its paragraphs. The internal consistency coefficient of the study tool was extracted, depending on the Cronbach's Alpha coefficient for each of the study variables in all its dimensions. It is a percentage that exceeds the minimum acceptable level for the purposes of scientific research, which is $(70 \%)$, and the following Table 1 shows the values of the stability coefficient.

\subsection{Statistical manipulations}

After data were entered using the SPSS.22.1, the following statistical treatments were used,

1- Descriptive statistic measures to describe the characteristics of the study population in terms of numbers, percentages, arithmetic averages, and standard deviation.

2- Multiple regression analysis to test the validity of the study model, and the effect of the independent variable on the dependent variable.

3- Stepwise Multiple Regression Analysis to test the entry of independent variables into the dependent variable prediction equation.

4- Cronbach's Alpha equation for internal consistency to verify the stability of the study tool.

5- Using the AMOS analysis program, based on the Path Analysis test, and the paths are represented by the independent, dependent and intermediate variables.

\section{Previous studies}

Stephen et al. (2019) conducted a study aimed at demonstrating the impact of entrepreneurial marketing practices on the competitive advantage of small and medium enterprises in Enugu state, Nigeria. The field of study was concentrated on small and medium enterprises (SMEs) in Enugu State, Nigeria. The study population included all employees of small and medium enterprises, managers and owners, in Enugu state in Nigeria, while the study sample included (356) small and medium enterprises in Enugu state in Nigeria. The descriptive analytical approach was used to achieve the objectives of the study, and the questionnaire was used as a main tool to test the study's hypotheses. After conducting the necessary statistical analyzes, the study reached several results, most notably that the practice of focusing on opportunities positively affects the competitive advantage of small and medium enterprises in Enugu state in Nigeria. Hoque et al. (2019) conducted a study aimed at demonstrating the impact of entrepreneurial marketing on the performance of Bangladeshi SMEs, in addition to identifying the role of organizational culture in the relationship between entrepreneurial marketing and the performance of Bangladeshi SMEs. The field of study shall be SMEs in Bangladesh. The study population included all employees of small and medium-sized Bangladeshi companies, while the study sample consisted of (384) owners of a small and medium-sized Bangladeshi company who were selected in a cluster random manner. The descriptive analytical approach was used to achieve the objectives of the study, and the questionnaire was used as a main tool to test the study's hypotheses. After conducting the necessary statistical analyzes, the study reached several results, most notably the existence of an impact of entrepreneurial marketing on the performance of Bangladeshi SMEs, and that organizational culture plays a moral mediating role in the relationship between entrepreneurial marketing and the performance of Bangladeshi SMEs. Lettelton (2018) conducted a theoretical study focused on distinguishing between supply and demand strategies for strategic intelligence, and the study concluded that the adoption of business intelligence policy in business organizations directly affects creativity, and that the support of senior management to apply business intelligence effectively contributes to creating a creative environment The study reached results, the most important of which were: the necessity of adopting the concept of business intelligence in organizations and in all its dimensions for its important and effective role in achieving creativity in the organization, and encouraging creativity contributes positively to the organization's excellence and achieving more competitive advantages.

\subsection{What distinguishes the current study?}

Most of the previous studies focused on the importance of studying digital entrepreneurship, digital governance, and effective governance, as the study (Al-Barashdiya, 2021) focused on the role of the Corona pandemic in reshaping the entrepreneurship sector. The study by Al-Nusour (2020) also addressed the impact of applying digital Entrepreneurship on organizational performance in companies operating in the pharmaceutical industries within the perspective of social network theory. In Palestine, the study (Stephen et al., 2019) aimed to demonstrate the impact of entrepreneurial marketing practices on the competitive advantage of small and medium-sized enterprises in Enugu state, Nigeria. However, this study examined the impact of digital Entrepreneurship in its dimensions (digital creativity, digital leadership, digital knowledge) on digital supply chains in its dimensions (digital supply, digital marketing, digital services, digital logistics) individually and collectively, and the mediating role of business intelligence applications in the Jordanian hotel sector. As for this study, it dealt with the three variables (digital leadership, digital supply chains, and business intelligence applications) with some comprehensiveness that contributed modestly to enriching the field of study through the theoretical framework it will present that details the most prominent global experiences and models in managing and measuring digital Entrepreneurship and its impact In digital supply chains, and business intelligence applications. To highlight digital Entrepreneurship and its development and development mechanisms, and to identify the most prominent scientific methods and methods that help achieve this in the Arab environment in general and Jordan in particular. In practice, the study was more varied and objective in its approach to the study community 
(the Jordanian hotel sector), and by relying on an appropriate research sample, which gives the possibility to generalize the results of this study and benefit from them, and according to the researcher's knowledge that it is the only study in this field that addressed these dimensions, and this is what distinguishes the study from its predecessors.

\subsection{Hypothesis testing}

Before applying the regression analysis to test the hypotheses, some tests were performed to ensure that the data fit the assumptions of the regression analysis, as follows: With regard to the assumption that there should be no high correlation between the independent variables "Multicollinearity", the researcher performed the Variance Inflation Factor VIF, and Tolerance test for each of the independent variables. Table 2 indicates that if the variance inflation factor (VIF) for the variable exceeds (10) and the permissible variance value is less than (0.05), then it can be said that this variable has a high correlation with other independent variables and thus will lead to a problem in regression analysis. This rule has been relied on to test the "Multicollinearity" between the independent variables. As indicated in Table 2, which contains the independent variables, the value of the variance inflation factor (VIF) and the permissible variance "Tolerance" for each variable. We note that the value of (VIF) for all the variables included in the first main hypothesis was less than (10) and ranged (5.102-2.789). There is no high correlation (multicollinarity) between the dimensions of the independent variable. It was confirmed that the data follow a normal distribution by calculating the Skewness coefficient, where the values were less than (1), and the validity of the model was confirmed to test the main and dependent hypotheses of the study.

Table 2

Test the variance inflation factor, the permissible variance, and the skew modulus

\begin{tabular}{lccc}
\hline Variables & Variables & $\begin{array}{c}\text { Tolerance (greater than } \\
\mathbf{5 \%})\end{array}$ & $\begin{array}{c}\text { Contrast coefficient (less } \\
\text { than 10) }\end{array}$ \\
& & $\begin{array}{c}\text { Sprains } \\
\text { (less than 1) } \\
\text { Skewness }\end{array}$ & 0.395 \\
\hline The first main hypothesis & digital creativity & 0.211 & 5.102 \\
& digital leadership & 0.287 & 2.789 \\
\hline
\end{tabular}

In order to investigate the assumption of the normal distribution of the data, it was based on calculating the value of the Skewness coefficient for the variables, and as Table 2 indicates, the value of the skewness coefficient for all study variables was less than (1). Therefore, it can be said that no There is a real problem related to the normal distribution of the study data, and the validity of the model will be confirmed for each hypothesis separately.

Table 3

The results of the analysis of variance to ensure the validity of the model to test the hypotheses of the study

\begin{tabular}{|c|c|c|c|c|}
\hline Independent variable & Dependent variable & R2 modified & Calculated $\mathrm{F}$ value & $\mathrm{F}$ significance level \\
\hline The first main premise: & Digital supply chains & 0.599 & $88.082 *$ & 0.000 \\
\hline \multirow[t]{4}{*}{ digital entrepreneurship } & Digital supply & 0.521 & $49.603^{*}$ & 0.000 \\
\hline & Digital marketing & 0.431 & $44.735^{*}$ & 0.000 \\
\hline & Digital services & 0.473 & $47.019^{*}$ & 0.000 \\
\hline & Digital logistics & 0.492 & $61.456^{*}$ & 0.000 \\
\hline
\end{tabular}

* Statistically significant at the level $(\alpha \leq 0.05)$.

Table 3 shows the validity of the study's hypotheses testing model, and given the high value of (F) calculated from its tabular value, as the dimensions of digital Entrepreneurship explain (59.9\%) of the variance in the total dependent variable (digital supply chains), as it is also explained (52.1\%) of the variance in the dimension (digital supply), and the dimensions of digital Entrepreneurship explained $(43.1 \%)$ of the variance in the dimension of (digital marketing), and the dimensions of digital Entrepreneurship explained $(47.3 \%)$ of the variance in the dimension (digital services). Finally, the dimensions of digital Entrepreneurship explained (49.2\%) of the variance in the dimension (digital logistics).

$\mathrm{H}_{\mathrm{ol}}$ : There is no statistically significant effect at the level of significance $(\alpha \leq 0.05)$ for digital entrepreneurship with its dimensions (digital creativity, digital leadership, digital knowledge), in the digital supply chains with its dimensions (digital supply, digital marketing, digital services, digital logistics) in the sector Jordanian hotels.

\section{Table 4}

Results of multiple regression analysis to test the impact of digital Entrepreneurship on the digital supply chain in the Jordanian hotel sector

\begin{tabular}{|c|c|c|c|c|c|}
\hline Digital Entrepreneurship & $\mathrm{B}$ & Standard & Beta & calculated t value & t. significance level \\
\hline digital creativity & 0.234 & 0.050 & 0.271 & $5.861^{*}$ & 0.000 \\
\hline digital leadership & 0.356 & 0.045 & 0.380 & $8.893^{*}$ & 0.000 \\
\hline digital knowledge & 0.169 & 0.047 & 0.206 & $4.527 *$ & 0.000 \\
\hline
\end{tabular}

* Statistically significant at the level $(\alpha \leq 0.05)$

It is evident from the statistical results presented in Table 4, and from the follow-up of (t) test values that the following subvariables related (digital creativity, digital leadership, digital knowledge) have an impact on digital supply chains in the Jordanian hotel sector, calculated and amounted to 5.861, 8.893, 4.527, respectively, which are significant values at the level of 
significance $(\alpha \leq 0.05)$. The above requires the following: Rejection of the null hypothesis which states that there is no significant effect with statistical significance for the digital Entrepreneurship variables (digital creativity, digital leadership, digital knowledge) in the digital supply chains in the Jordanian hotel sector.

\section{Table 5}

Results of Stepwise Multiple Regression to predict digital supply chains through the dimensions of digital Entrepreneurship as independent variables

\begin{tabular}{|c|c|c|c|}
\hline The order of entry of the independent elements in the prediction equation & $\begin{array}{l}\text { R2. value } \\
\text { Modified }\end{array}$ & The calculated $t$ value & denotation level * \\
\hline digital leadership & 0.511 & $9.197 *$ & 0.000 \\
\hline digital creativity & 0.573 & 4.984* & 0.000 \\
\hline digital knowledge & 0.599 & $3.930 *$ & 0.000 \\
\hline
\end{tabular}

* Statistically significant at the level $(\alpha \leq 0.05)$

When conducting a Stepwise Multiple Regression analysis to determine the importance of each independent variable in contributing to the mathematical model, which represents the impact of digital Entrepreneurship (digital innovation, digital leadership, digital knowledge) on the digital supply chains in the Jordanian hotel sector. As can be seen from Table 5, which shows the order of entry of the independent variables into the regression equation, the digital leadership variable explains the amount of $(51.1 \%)$ of the variance in the dependent variable, and the entry of the digital creativity variable is explained with the digital Entrepreneurship variable $(57.3 \%)$ of the variance in the dependent variable, and finally entered the digital knowledge variable, where it was explained with the previous two variables $(59.9 \%)$ of the variance in the digital supply chains in the Jordanian hotel sector as a dependent variable. This result means that Jordanian hotels pay sufficient attention to digital Entrepreneurship to provide employees with new experiences and knowledge to reach a state of cognitive equilibrium that provides them with performance skills to improve performance, enhance organizational capabilities and enable individuals to acquire skills and information so that they are able to cope with current and future challenges and qualify them, through Optimal use of the available material and human resources in order to bring about change and development and improve the performance of individuals at work, and encourage them to exert the greatest possible effort and effort. This result may also be attributed to the fact that the hotels researched apply policies and programs that help develop the administrative skills possessed by individuals, and provide a certain amount of Freedom to participate in the administrative process, and these policies serve as a system that contains initiatives, approaches and tools directed to create an ideal flow of knowledge to achieve organizational goals efficiently and effectively, by reducing cost, increasing productivity, achieving excellence in business performance, and enhancing the organization's ability to maintain and improve organized performance based on experience and knowledge. This reflects the importance of achieving the dimensions of digital Entrepreneurship on digital supply chains, or what is known as the main processes of various organizations and institutions, providing a vision for the organization's future visions, designing its mission, and defining its long-term goals, and determining the dimensions of the expected relationships between it and its environment, thus contributing to the statement of the surrounding opportunities and risks. and its distinctive strengths and weaknesses, with the aim of making strategic decisions affecting the long-term, reviewing and evaluating them to achieve the goals of the organization efficiently and effectively. Perhaps you may return to the belief that achieving the dimensions of digital leadership is the responsibility of the central body, which is the mother hotel to which the hotel branches belong, and that the role of these branches is limited to implementation and carrying out daily tasks entrusted to them.

$\mathrm{H}_{02}$ : There is no statistically significant effect at the level $(\alpha \leq 0.05)$ of digital Entrepreneurship with its dimensions (digital creativity, digital leadership, digital knowledge) on digital supply chains with its combined dimensions through business intelligence applications in the Jordanian hotel sector.

In order to test this hypothesis, the researcher conducted a path analysis of the hypothetical variables or the so-called Structural Equation Modeling. The idea of this analysis is based on the generation of a set of hypothetical variables (Latent Variables) through a larger group of real variables (Observed Variables), Where we assume that these default variables affect a set of real variables through the so-called factor loading, and then a path analysis using latent variables is performed for those new default variables that have been generated. The AMOS.25 program has been used. to perform this analysis. The results of the analysis came as in Table No. (15), where the chi-square value was (6.85) and a probability value (0.000), which is significant at the level of (0.05), and this means that there are significant differences between the variance and covariance matrices due to the sample size, as well It is noted that the value of the Goodness Fit Index reached (0.945), and the value of this index is equal to the correct one for the Perfect Model. The value of the Adjusted Goodness Fit Index has reached (0.967), and the value of this indicator is the upper limit of the perfect model, and one is correct, but its minimum can be less than zero. As for the Normed Fit Index, its value was (0.961). This indicator indicates that the models whose value is less than (0.900) need to make adjustments, and with regard to the Comparative Fit Index, its value is (0.952) and this index ranges between (0 and 1$)$ and is close to one It should be noted that the values of this indicator range between $(0$ and 1$)$ and close to one for the appropriate model, and it is noted that the root mean square of the error approximation (RAMSEA) ) reached (0.03), which is a value ranging between $(0.05$ and 0.08$)$ and reflects an acceptable value for approximating the error, and it is worth noting that if the value of this indicator exceeds $(0.10)$, the model is considered unacceptable. 
Table 6

Fit value indicators

\begin{tabular}{ccc}
\hline Indications & Required value * & The actual value \\
\hline Relative chi-square (CMIN/DF) & $\geq 5$ & 6.815 \\
Quality of Validity (GFI) & $0.90 \leq$ & 0.945 \\
Modified Quality of Validity (AGFI) & $0.80 \leq$ & 0.967 \\
Standardized Validity Index (NFI) & $0.90 \leq$ & 0.961 \\
Comparative Validity Index (CFI) & $0.90 \leq$ & 0.952 \\
Incremental Validity Index (IFI) & $0.90 \leq$ & 0.924 \\
Root mean square error of approximation (RMSEA) & 0.08 to 0.05 & 0.03 \\
\hline
\end{tabular}

It is also noted from Table 6 that the direct impact of digital Entrepreneurship on digital supply chains is (2.615), which is statistically significant at the level $(\alpha \leq 0.05)$, where the critical value of the path (Critical Ratio) reached (4.11) and a probability value (0.000), The direct impact of business intelligence applications on digital supply chains was (4.210), and this effect is statistically significant, as the critical value for this path was (3.65). Its probability value was $(0.002)$, and thus the indirect effect of digital Entrepreneurship on digital supply chains through business intelligence applications as an intermediate variable equals (13.21), and this indirect effect is significant at the level $(\alpha \leq 0.05)$, where the probability value (P) (0.000), Thus, we reject the fourth main hypothesis, which states: There is no statistically significant effect at the level $(\alpha \leq 0.05)$ of digital entrepreneurship with its dimensions (digital creativity, digital leadership, digital knowledge) on digital supply chains with its combined dimensions through business intelligence applications by their combined dimensions in the sector Jordanian hotels.

Table 7

The results of the path analysis test for the impact of digital Entrepreneurship on digital supply chains through business intelligence applications as an intermediate variable

\begin{tabular}{|c|c|c|c|c|c|}
\hline The path & Direct effect & Indirect effect & overall effect & Critical path value (CP) & $\mathrm{P}$ value \\
\hline Digital Entrepreneurship $\rightarrow$ Digital Supply Chains. & 2,615 & ---- & & 4.11 & 0.000 \\
\hline $\begin{array}{c}\text { Business Intelligence Applications } \rightarrow \text { Digital Supply } \\
\text { Chains }\end{array}$ & 4.210 & ---- & 2,615 & 3.65 & 0.000 \\
\hline $\begin{array}{l}\text { Digital Entrepreneurship } \rightarrow \text { business intelligence ap- } \\
\text { plications } \rightarrow \text { digital supply chains. }\end{array}$ & 2.20 & 11.01 & 4.210 & & 0.000 \\
\hline
\end{tabular}

This result explains that business intelligence applications affect digital supply chains, and therefore the increased interest in business intelligence applications would generate an impact in improving digital supply chains. This result can be explained by the fact that Jordanian hotels are keen to continuously improve their performance by formulating realistic and measurable goals to ensure their continuous achievement and following the scientific foundations to solve work problems, and using management concepts that seek growth, renewal, and excellence in the accomplishment of tasks and responsibilities, and the reason for this result Achieving the competitiveness of organizations is represented in the ability of these hotels to develop themselves and improve their work. Achieving competitiveness determines strengths and weaknesses, and helps hotels define digital supply chains, and these elements invest in adapting to environmental changes. Policies of improving digital supply chains help in the ability of these hotels to identify competing hotels by identifying weaknesses and strengths in their performance and trying to overcome them and enhance strengths, and reliance on filling some of their jobs to attract and attract individuals with experience and efficiency and encourage workers and strengthening them to develop self-control when performing the tasks required of them. This result also explains that Jordanian hotels can deal with appropriate strategic alternatives to implement and follow up on strategies, because there is an integrative and participatory overlap between strategy formulation and implementation that helps fill the expected deficiency in formulation. The effective implementation of the strategy requires that Jordanian hotels seek to find supportive viewpoints for the specific strategies and mobilize all human and material energies to complete the required work within the timetables set for them and without mistakes that may lead to the failure of this strategy through the development of workers' control over themselves in the performance of their work.

\section{Recommendations}

In light of the previous results, the study recommends the following:

1. The necessity of enhancing digital Entrepreneurship in Jordanian hotels by focusing on the holistic view of these hotels and their environment, whether the internal environment that focuses on strengths and weaknesses in the hotel's capabilities or their external environment that brings opportunities and challenges.

2. Take full advantage of the components of digital Entrepreneurship, such as directing digital creativity to study the internal and external environment of hotels, the extent to which they are affected by external variables, and how to hedge against potential external threats.

3. Maintaining and developing the hotel's digital supply chains in all its axes: digital supply, digital marketing, digital services, digital logistics, by promoting interest in digital Entrepreneurship and business intelligence applications, enhancing these capabilities and examining all the factors that contribute to improving digital supply chains. 
4. Enhancing business intelligence applications in Jordanian hotels through: The hotel management quickly reformulating strategies when market conditions require it and working to provide a set of strategic measures to face change, using production technology that allows a large number of operations to be conducted in a fast manner, increasing the number of modifications that Hotels conduct annual services on offering new products efficiently.

5. Carrying out advertising campaigns with the aim of influencing the tastes of customers and enhancing the position of hotels in the market to allow control over competitors, which hinders the entry of new competitors.

6. Conducting more future studies and testing other variables that the study did not address that motivate hotels to plan or practically implement digital supply chains.

\section{References}

Al-Barashdiya, H. S. A. (2021) Digital Entrepreneurship in the Light of the Corona Pandemic (Covid 19): Opportunities and Challenges. Journal of Information Studies \& Technology, 1, 259-284.

Al-Ghazawi, G. (2013) The use of business intelligence systems in the development of human capital, an exploratory study of a sample of employees in the Ministry of Health, Al-Ghari. Journal of Economic and Administrative Sciences, 9(28), 49-73.

Al-Tamari, S. S. S. (2017) The impact of business intelligence on building human resource management strategies, a field study in Jordanian commercial banks, an unpublished master's thesis. Amman Arab University, Jordan.

Al-Tarawneh, K. A., \& Shalash, A. I. (2015) The impact of perceived strategic supply management skills on the performance of supply management through supplier integration: a field study in Jordanian industrial companies operating in Sahab City. The Jordanian Journal of Business Administration, 11(1).

Asamoah, D., Nuertey, D., Agyei-Owusu, B., \& Akyeh, J. (2021). The Effect of Supply chain response on customer development. The International Journal of Logistics Management, Vol. Pre-print No. Pre-print. https://doi.org/10.1108/IJLM-032020-0133

Autio, E., Nambisan, S., Thomas, L. D., \& Wright, M. (2018). Digital affordances, spatial affordances, and the genesis of entrepreneurial ecosystems. Strategic Entrepreneurship Journal, 12(1), 72-95.

Collins, R.J. (2017). Better Business Intelligence. How do you know more about your competitors? Chalford: Management Books.

El Baz, J., Laguir, I., \& Stekelorum, R. (2019). Logistics and Supply Chain Management Research in Africa: a systematic literature review and research agenda. International Journal of Logistics Management, 30(1), 8-38.

Fuld, D. (2015). Operation Intelligence: A Management Checklist. Canadian Business Review, 18(2), 39-42.

Giannakis, M., Spanaki, K., \& Dubey, R. (2019). A cloud-based supply chain management: effects on supply chain response. Journal of Enterprise Information Management, 32(4), 585-607.

Hoque, A., Awang, Z., \& Gwadabe, U. (2019). The impact of entrepreneurial marketing on the performance of Bangladeshi SMEs and the role of organizational culture: structural equation modeling. Journal of Management and Operations Research, 1(16), 1-21.

Kumar, R., \& Singh, R. K. (2017). Coordination and Response Issues in Supply Chains for SMEs: A Review. Benchmarking: An International Journal, 24(3), 635-650.

Lettelton, P. (2018). Using Business Intelligence to Sustain Innovation. Technology Research Department, 11(9), 94-122.

Bach, M. P., Jaklič, J., \& Vugec, D. S. (2018). Understanding impact of business intelligence to organizational performance using cluster analysis: does culture matter. International Journal of Information Systems and Project Management, 6(3), 63-86.

Nithya, N., \& Kiruthika, R. (2021). Impact of Business Intelligence Adoption on performance of banks: a conceptual framework. Journal of Ambient Intelligence and Humanized Computing, 12(2), 3139-3150.

Nsour, B. (2020). The impact of digital leadership on organizational performance from the point of view of managers of Jordanian pharmaceutical companies within the perspective of social network theory. The Jordanian Journal of Business Administration, 16, pp. 259-284.

Shen, B., Xu, X., \& Guo, S. (2019). The impacts of logistics services on short life cycle products in a global supply chain. Transportation Research Part E: Logistics and Transportation Review, 131, 153-167.

Stephen, O. U., Ireneus, N., \& Moses, O. C. (2019). Entrepreneurial marketing practices and competitive advantage of small and medium size enterprises in Nigeria. European Journal of Business and Innovation Research, 7(3), 1-30.

Younis, F. (2015) The impact of the integrative role of the business intelligence system and knowledge transfer processes in building the learning organization Field research in Korek Mobile Communications Company in the city of Baghdad. Journal of Economic and Administrative Sciences, 21(86), 478.

Zhu, Z., \& Lin, S. F. (2019). Understanding entrepreneurial perceptions in the pursuit of emerging e-business opportunities: The dimensions and drivers. Computers in Human Behavior, 95, 252-261.

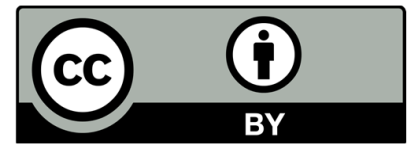

(C) 2022 by the authors; licensee Growing Science, Canada. This is an open access article distributed under the terms and conditions of the Creative Commons Attribution (CC-BY) license (http://creativecommons.org/licenses/by/4.0/). 